Open Access

\title{
Assessing Primary Care Physician's Beliefs and Attitudes of Asthma Exacerbation Treatment and Follow-Up
}

\author{
William Lincourt ${ }^{*}, 1$, Richard H. Stanford ${ }^{1}$, Alicia Gilsenan ${ }^{2}$, Dana DiBenedetti ${ }^{2}$ \\ and Hector Ortega ${ }^{1}$
}

\author{
${ }^{I}$ GlaxoSmithKline, Research Triangle Park, NC, USA \\ ${ }^{2}$ RTI Health Solutions, Research Triangle Park, NC, USA
}

\begin{abstract}
Objective: The objective of this survey was to assess adult primary care physicians' and pediatricians' perceptions of asthma exacerbation management, including beliefs concerning the discharge of patients from the emergency department (ED) following asthma exacerbations.
\end{abstract}

Methods: This was a cross-sectional survey of primary care physicians (PCPs) treating adult or pediatric patients. Surveys were mailed to physicians and included questions on how PCPs define an exacerbation, how they are notified and how they followed-up with their patients who experienced exacerbations.

Results: A total of 189 physicians were targeted in this survey, with $124(65 \%)$ returning a completed survey. The majority of physicians agreed that an exacerbation included worsening asthma requiring a course of oral corticosteroids $(83 \%)$. However, $\geq 70 \%$ of physicians agreed that an exacerbation could also include events which did not require OCS. Overall, $71 \%$ of PCPs believed that the majority of their patients' asthma exacerbations were treated in the doctor's office with only $6 \%$ believing the majority were treated in the ED. Over $90 \%$ of PCPs surveyed said they scheduled a follow-up with their patients "all or most of the time" when notified of an ED visit for an asthma exacerbation. Of the adult PCPs surveyed, 20\% said they were never notified when one of their patients received treatment in the hospital because of an asthma exacerbation, whereas only $10 \%$ of pediatricians said they were never notified. The majority of PCPs surveyed (79\%) indicated that if a controller medication was warranted, the ED staff should initiate treatment at time of discharge.

Conclusions: This study showed that healthcare providers may not share a common definition of an asthma exacerbation. In addition, most physicians believe that the majority of exacerbations are treated in their office or at home. Further, most agreed that if a controller medication was warranted, the ED or urgent care staff should initiate treatment.

Keywords: Asthma, exacerbations, emergency department, primary care.

\section{INTRODUCTION}

Asthma exacerbations are often associated with lack of asthma control. They can be severe enough to lead to emergency department (ED) visits or hospital admissions, and even death [1]. Patients who have exacerbations requiring an $\mathrm{ED}$ visit, hospitalization, or intensive care unit admission are at greater risk of exacerbations in the future. In addition, severe asthma exacerbations can occur in patients across the spectrum of disease severity including patients considered to have "mild" asthma [2-4]. Asthma guidelines indicate that disease awareness education, follow-up care and assessment of appropriate use of long-term controller medications should be priority items in the discharge planning of patients from the ED or hospital [1]. In a study by Singh and colleagues, $31 \%$ of patients overall used the ED first when experiencing asthma problems, and $22 \%$ reported the $\mathrm{ED}$ as their usual source of asthma prescriptions. Even in patients with a PCP, $10 \%$ used the ED as their primary provider of asthma medications [5].

*Address correspondence to this author at the GlaxoSmithKline, Five Moore Drive, MS 17.1315F.1C, Research Triangle Park, NC 27709-3398, USA; Tel: (919) 483-6284; Fax: (919) 483-4300;

E-mail: william.r.lincourt@gsk.com
Importantly, studies have shown that approximately $70 \%$ of patients do not receive controller medications, such as inhaled corticosteroids, on discharge from the $\operatorname{ED}[6,7]$. The reason for this is not well understood. It may be that ED staff focuses solely on treating the current acute event or physicians assume patients will receive these medications upon follow-up with their PCP.

Therefore, we sought to assess adult primary care physician and pediatrician perceptions of asthma exacerbation management, including beliefs concerning the discharge of patients from the ED following asthma exacerbations.

\section{METHODS}

\section{Study Design}

This was a cross-sectional survey of PCPs, conducted from June through August 2008. The final survey was approved by the institutional review board of RTI International.

\section{Study Setting and Population}

Physicians from thirty-five adult primary care and 29 pediatric study sites focusing on the delivery of health care that were currently participating in an ongoing observational 
study were invited to complete the questionnaire. Sites were identified from a proprietary database of more than 2000 US physicians with membership in the Primary Care Network, an organization that provides continuing medical education to its members. Study sites were excluded if they specialized in asthma treatment or had an asthma specialist on staff, or if they had participated in a respiratory-related clinical research study in the previous 3 years.

\section{Study Protocol and Measurements}

This survey was administered to physicians as part of the the Asthma Control Characteristics and Prevalence Survey Studies (ACCESS; ADA111118/19). Although physicians from across the country participated in this exacerbation survey it was not the intent to get a geographic representation or random sample of physician responses and we did not stratify results by region or practice dynamics (e.g. size of practice).

A brief questionnaire was developed by RTI Health Solutions in collaboration GlaxoSmithKline, a maker of inhaled asthma medications, which included questions on how PCPs define and are informed of their patients' exacerbations, and how they followed-up with patients who experienced exacerbations. Initially, a pilot survey was conducted to cognitively pre-test the questions among physicians (5 PCPs and 5 pediatricians) and to identify any revisions necessary to maximize understanding and ease of completion of the final survey. Based upon feedback from the pilot, modifications were made to the final survey instrument. These changes were minor and included rewording of several questions to increase understanding. The final survey contained a total of 25 questions, 18 of which dealt with issues related to asthma exacerbations (Appendix I) and are described in this report Exacerbations in this survey were identified from a spectrum of options by the physicians such as the need for hospitalization, use of oral corticosteroids, ED visits, increase in rescue medication use; or change in controller medication without a course of oral corticosteroids.

\section{Data Analysis}

Descriptive analysis and tables summarizing survey results were produced for the entire sample and also stratified by type of PCP (adult or pediatric). For continuous-type data, the mean, standard deviation, median, minimum, and maximum were generated, whereas counts and percentages were produced for categorical data. Data analysis was performed using SAS version 9.1.3 (SAS Institute Inc., Cary, NC).

\section{RESULTS}

A total of 189 physicians were sent the final survey, with $124(65 \%)$ returning a completed survey. Among the 124 respondents, there were 56 adult primary care physicians and 68 pediatricians. This sample of physicians was geographically dispersed across the US (25\% Midwest, $22 \%$ Northeast, 25\% South, and 28\% West).

The majority of physicians agreed that an exacerbation is defined as worsening asthma requiring a course of oral corticosteroids (83\%). However, $\geq 70 \%$ of physicians agreed that an exacerbation could also include events which did not require OCS, but rather, resulted in increased use of rescue albuterol for two or more consecutive days or required an inhaled or nebulized albuterol intervention in the physician's office or prompted the need to change maintenance medications (Fig. 1). When asked to rank events most likely to trigger an exacerbation, both adult and pediatric PCPs ranked poor compliance with asthma medication, common cold or flu or allergen exposures the highest. Pediatric PCPs were more likely to rank common cold or flu as the primary trigger while adult PCPs were more likely to rank poor compliance with asthma medication as the primary trigger. When asked about which season Adult PCPs see the most asthma exacerbations, $45 \%$ reported winter, 30\% reported fall, $23 \%$ reported spring, $0 \%$ reported summer, and $5 \%$ reported they do not see any seasonal differences. When Pediatricians were asked the same question, 48\% reported fall, 39\% reported winter, 13\% reported spring, $0 \%$ reported summer, and $0 \%$ reported they do not see any seasonal differences.

Overall, $71 \%$ of All PCPs believed that the majority of their patients' asthma exacerbations were treated in the doctor's office. Further, 19\% believed treatment usually occurred in the patient's home, $6 \%$, the ED, another $4 \%$ in urgent care, and 3\% said they did not know. Approximately half of the PCPs $(55 \%$ of adult PCPs and $43 \%$ of pediatricians) said they were notified of their patient's ED visit or hospitalization within a few days of the event, whereas $27 \%$ and $49 \%$, respectively, were notified at the time of hospital visit. Only $4 \%$ of adult and $10 \%$ of pediatric PCPs were notified of an urgent care visit at the time of the event. Approximately $20 \%$ of adult and $10 \%$ of pediatric PCPs said they are never notified of an ED visit for asthma and became of aware of these events later (Table 1). On the other hand, results show that a large percentage $(40 \%)$ of adult PCPs said they receive no notification of their patient's visit to an urgent care center for an exacerbation; this percentage is less $(29 \%)$ for pediatric providers. When adult PCPs and pediatricians were notified that their patient had been treated for an asthma exacerbation in the ED, the majority $(91 \%)$ required a follow-up visit in the office "all of the time or most of the time". In contrast, only $73 \%$ stated that they followed-up through an in-office visit for patients who treated an exacerbation at home. The majority of physicians surveyed (79\%) indicated that if a controller medication was warranted, the ED or urgent care staff should initiate treatment at time of discharge.

Table 1. How Primary Care Physicians are Notified of ED or Hospital Admissions for Asthma Exacerbations

\begin{tabular}{|l|c|c|}
\hline \multicolumn{1}{|c|}{ Method of Information* } & Adult PCPs & Pediatricians \\
\hline \hline Call from the ED & $13(23.2 \%)$ & $24(35.3 \%)$ \\
\hline $\begin{array}{l}\text { Note, letter, fax or e-mail } \\
\text { from the ED }\end{array}$ & $33(58.9 \%)$ & $43(63.2 \%)$ \\
\hline $\begin{array}{l}\text { Call from hospital or } \\
\text { attending physician }\end{array}$ & $3(5.4 \%)$ & $20(29.4 \%)$ \\
\hline $\begin{array}{l}\text { Contact from patient via phone, } \\
\text { e-mail or office visit }\end{array}$ & $17(30.4 \%)$ & $33(48.5 \%)$ \\
\hline Do not get notified & $11(19.6 \%)$ & $7(10.3 \%)$ \\
\hline Other & $0(0 \%)$ & $2(2.9 \%)$ \\
\hline *Sum totals may exceed 100\% as respondents were allowed to select all that applied.
\end{tabular}




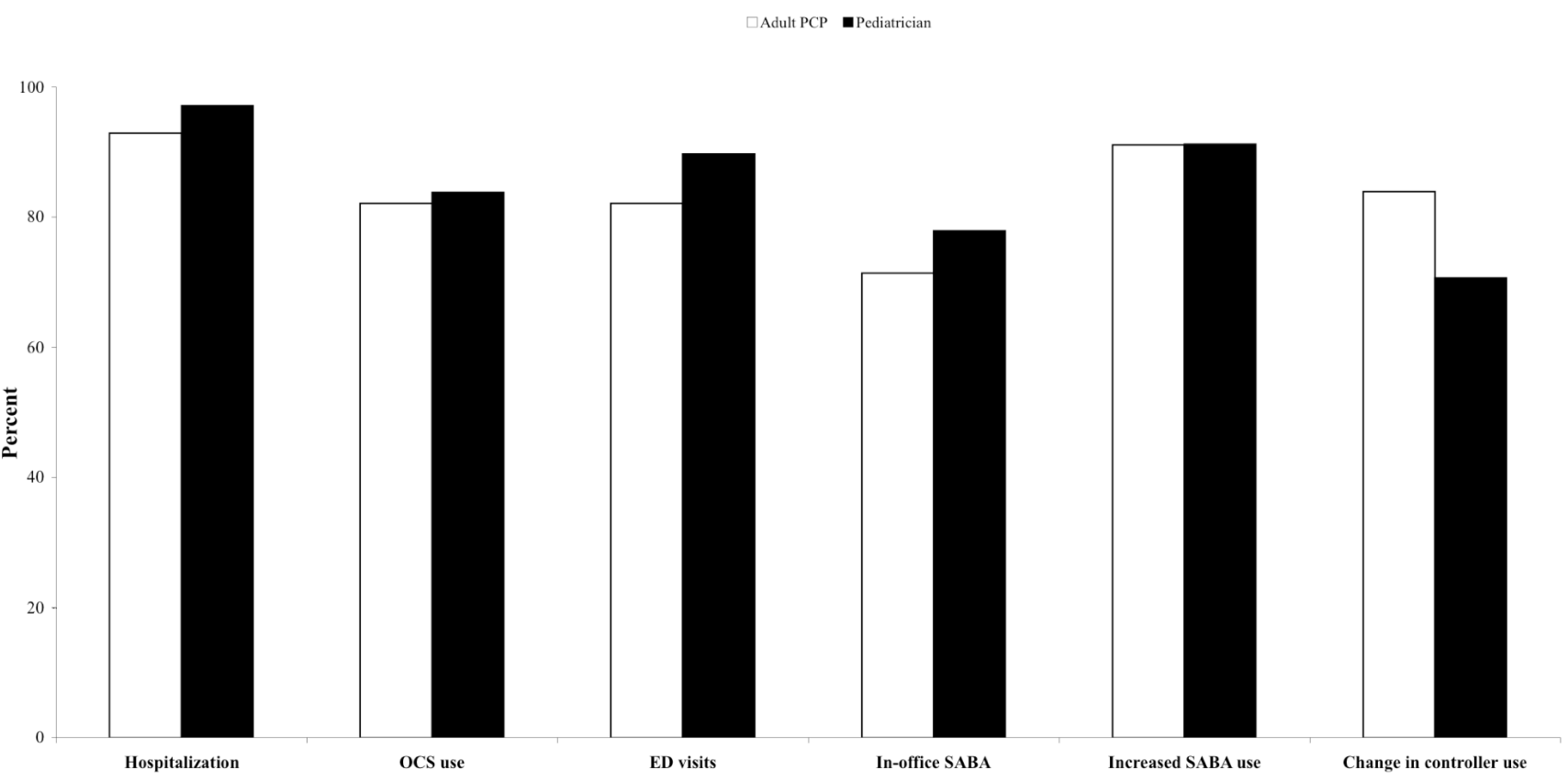

Fig. (1). Percent of physicians that agree an asthma exacerbation had occurred when different definitions were provided.

\section{DISCUSSION}

Exacerbations consist of a sustained, often progressive, deterioration in asthma symptoms and airflow obstruction that occurs over hours to days and can last for days to weeks, where additional medications and/or emergency care is often needed. These attacks generally allow time for intervention; however, a few patients have a rapid onset or a severe asthma exacerbation and require immediate medical attention [8]. In this survey most PCPs reported that the majority of their patient's asthma exacerbations were treated in the doctor's office. These findings suggest that the intensity of the exacerbations could have been perceived as not too severe and therefore, required a less aggressive intervention. This could be also a function of the interpretation or definition of an exacerbation. In 2008 the National Health Interview Survey (NHIS) included the following question to their asthma prevalence survey, "During the past 12 month months, have you had an episode of asthma or an asthma attack?" [9]. The use of the term "asthma attack" appears to be in recognition of a broader definition of exacerbations that may be less severe but still clinically important. Considering the significant impact these "asthma attacks" have on the patient and the need for a clinical response, perhaps the primary care physician's definition is also appropriate with the modifier of mild or moderate exacerbation. The burden of uncontrolled asthma may be under reported considering that these milder events may not be captured in many epidemiological surveys that track asthma morbidity. Therefore, a broader, common definition of an asthma exacerbation between all health care providers may help the precision for reporting serious episodes of worsening asthma and in turn, increase quality of care for millions of patients with asthma. In addition, patients seen in the sites surveyed may have been more likely not to use the ED for less severe events or for events that occurred during normal office hours.
An interesting difference between responses of adult PCPs and pediatricians was the season when providers observe the greatest number of asthma exacerbations. For adult PCPs, the majority reported winter as being the season when they observe the most exacerbations in their patient population. On the other hand, the majority of pediatricians reported fall as the season when they observe the greatest number. This difference may in fact relate to the start of school in younger asthmatics.

PCPs questioned in this survey stated they were usually notified within a few days of an ED visit in the majority of cases, with pediatricians reporting being notified $90 \%$ of the time. This number may be an over-estimate since it represents only the events the PCP was made aware of at a later date. Over $90 \%$ of PCPs surveyed said they scheduled a follow-up with their patients "all or most of the time" when notified of an ED visit for an asthma exacerbation. Also evident from the data collected was that PCPs are less aware of urgent care visits for asthma than for ED visits or hospitalizations. These results highlight the importance of having consistent communication to allow follow-up with patients in a timely manner and possibly preventing further asthma morbidity.

The majority of physicians surveyed $(79 \%)$ indicated that, if a controller medication was warranted, the ED or urgent care staff should initiate treatment at time of discharge. National guidelines for the treatment of asthma suggest that sufficient medication be prescribed so that patients may continue treatment until follow-up [1]. Despite the current evidence supporting the role of anti-inflammatory controller agents in the management of chronic asthma, data suggest that few ED physicians prescribe these agents at the time of discharge, perhaps thinking that the patient's primary care physician will address this at follow-up [10]. However, many patients may not see their primary care physician soon enough after an exacerbation, and even when they do, many 
do not receive inhaled corticosteroids. Also, for many patients with asthma without a PCP, the ED may be their primary source of care [5]. Patients who present to the ED with acute asthma are often under-treated prior to the event, or are non-adherent to their asthma management plan $[5,6]$. This was confirmed as most PCPs in our study identified poor medication compliance as a significant trigger for exacerbations. These patients may benefit from a reassessment of their asthma management plan, including starting controller therapy, since it has been found that inhaled corticosteroids initiated at ED discharge were most often continued at follow-up by the primary care physician [11]. Knowing that the majority of PCPs felt it was acceptable for a controller medication to be initiated in the ED may help remove one of the obstacles and perception associated with prescribing asthma controller medications at discharge. To facilitate continuity of care, ED physicians should also consistently provide summaries of care received and medications prescribed to the patient's PCP.

\section{LIMITATIONS}

There are some limitations to the present study design and survey methodology. The overall sample was geographically diverse with a response rate of over $60 \%$, however physicians that responded to the survey may not have been fully representative of overall PCP population of the US Due to the small sample size within each region, we were not able to stratify results by region or practice dynamics (e.g. size of practice) so it is unknown if any differences exist.

Another potential limitation is that the survey was qualitative in nature and written to elicit opinions of the physician based on physician experience and recall. Therefore, further studies would need to be conducted to quantify these findings.

\section{CONCLUSIONS}

This study showed that healthcare providers may not share a common definition of an asthma exacerbation. In addition, most physicians believe that the majority of exacerbations are treated in their office or at home. Further, most agreed that if a controller medication was warranted, the ED or urgent care staff should initiate treatment.

\section{ACKNOWLEDGEMENT}

The survey results reported in this article were funded by GlaxoSmithKline.

\section{APPENDIX 1}

\section{Part II - Asthma Exacerbations}

Questions 6-18 Present Various Scenarios Describing Exacerbations in the General Asthma Population

Please consider the following examples of controller medications when answering questions 6-18.

Inhaled Corticosteroids: Treat airway inflammation; for example, Flovent (fluticasone propionate), QVAR (BDP). Daily long-term oral systemic corticosteroid is a controller only in the case of severe persistent asthma.
Inhaled Cromolyn sodium and nedocromil: Stabilize mast cells; for example, Tilade (nedocromil).

Immunomodulators: Monoclonal antibody that prevents binding of $\operatorname{IgE}$ (anti-IgE) to the highaffinity receptors on basophils and mast cells; for example, Xolair (omalizumab).

Leukotriene modifiers: Include LTRAs and a 5lipoxygenase inhibitor; for example, Singulair (montelukast), Accolate.

LABAs: Bronchodilators with duration of effect of at least 12 hours; for example, Serevent (salmeterol) and Foradil (formoterol). Currently recommended in combination with an ICS.

Methylxanthines: Sustained-release theophylline with a mild to moderate bronchodilator effect; for example, Theo-Dur, Uniphyl.

Note: Reliever medications such as Ventolin (albuterol) are not considered controller medications.

To What Extent Do You Agree that Each Statement Below Describes an Asthma Exacerbation? For Each Statement, Please Choose One Answer Only

6. An exacerbation of asthma has occurred if there are significant respiratory symptoms requiring a course of oral corticosteroids (OCS) ( $\geq 5$ days) to control symptoms.

Agree

Neither agree nor disagree

Disagree

7. An exacerbation of asthma has occurred if significant symptoms require any change in asthma controller medication (e.g., change to different medication, increase in same medication, addition of new controller medication) without a course of OCS.

Agree

Neither agree nor disagree

Disagree

8. An exacerbation of asthma has occurred if an emergency room (ER) visit is required for asthma.

Agree

Neither agree nor disagree

Disagree

9. An exacerbation of asthma has occurred if in-patient hospitalization is required for asthma.

Agree

Neither agree nor disagree

Disagree

10. An exacerbation of asthma has occurred if an office visit for inhaled/ nebulized short-acting beta ${ }_{2}$ agonist (SABA) treatment is required, without requiring treatment with OCS.

Agree 
Neither agree nor disagree

Disagree

11. An exacerbation of asthma has occurred if increasing asthma symptoms require increased SABA use (e.g., more than 3 to 4 times daily) for 2 or more consecutive days.

Agree

Neither agree nor disagree

Disagree

12. In your experience, which of the following events are most likely to trigger an asthma exacerbation? Rank each event, with 1 being the most common trigger.

- Poor compliance with asthma therapy

- Smog or pollution

- Changes in air temperature or humidity

- Allergen exposure (all types)

- Smoking or second hand smoke

- Common cold or flu (viral infection)

- Strenuous physical activities

- Stress

- Hormonal events (e.g., birth control, menstrual cycle)

- Other, specify:

- Other, specify:

13. During the past 12 months, in what season have you observed the greatest number of asthma exacerbations in your respiratory patients? Choose one answer only.

Spring

Summer

Fall

Winter

No seasonal differences

14. In your opinion, where are the majority of your patients' asthma exacerbations treated?

ER

Urgent care clinic

Patient's home

Doctor's office

Other, specify:

Don't know or unable to determine

15. When you are notified about a patient's asthma exacerbation that was treated in the ER or hospital, what are the typical methods in which you are notified? Check all that apply.

Call from the ER

Note, letter, fax, or e-mail from the ER

Call from the hospital or attending physician or on-call physician
Do not usually get notified

Contact from patient via telephone, e-mail, or office visit

Other, specify:

16. When you are notified about a patient's asthma exacerbation that was treated in an urgent care clinic, what are the typical methods in which you are notified? Check all that apply.

Call from the urgent care clinic

Note, letter, fax, or e-mail from the urgent care clinic

Call from the on-call physician

Do not usually get notified

Contact from patient via telephone, e-mail, or office visit

Other, specify:

17. For patients you know had an ER visit or were hospitalized for an asthma exacerbation, how long after the ER visit or hospitalization are you typically notified about a patient's asthma exacerbation? Choose one answer only.

At time of visit/admission

A few days later

Within a month

Greater than 1 month

Other, specify:

18. For patients you know have visited an urgent care clinic for an asthma exacerbation, how long after the urgent care visit are you typically notified about a patient's asthma exacerbation? Choose one answer only.

At time of visit

A few days later

Within a month

Greater than 1 month

Other, specify:

\section{This Last Section Includes Questions About Treatments} for Asthma Exacerbations

19. How do you typically follow up with patients you know have visited the ER or urgent care for an asthma exacerbation? Check all that apply.

Telephone consultation with the patient

In-office visit

Other, specify:

Do not usually follow up unless they want to be seen

20. How do you typically follow up with patients you know have self-treated their asthma exacerbation? Check all that apply.

Telephone consultation with the patient In-office visit 
Other, specify:

Do not usually follow up unless they want to be seen

21. If you are notified that one of your patients has been treated for an asthma exacerbation in the ER, how often do you require a follow-up visit in your office? Choose one answer only.

All of the time

Most of the time

Some of the time

A little of the time

22. If your patient warrants a controller medication, is it appropriate for the ER staff to start the patient on the controller medication?

Yes

No

23. If your patient warrants a controller medication, is it appropriate for the urgent care staff to start the patient on the controller medication?

Yes

No

\section{REFERENCES}

[1] National Asthma Education and Prevention Program (NAEPP). Guidelines for the diagnosis and management of asthma. Expert Panel Report 3. Bethesda, MD: National Institutes of Health,
National Heart, Lung, and Blood Institute. NIH Publication 08 4051, July 2007.

[2] Adams RJ, Fuhlbrigge A, Guilbert T, Lozano P, Martinez F. Inadequate use of asthma medication in the United States: results of the asthma in America national population survey. J Allergy Clin Immunol 2002; 110: 58-64.

[3] Eisner MD, Lieu TA, Chi F, Capra A, Mendoza G, Blanc PD. Beta agonists, inhaled steroids, and the risk of intensive care unit admission for asthma. Eur Respir J 2001; 17: 233-40.

[4] Lieu TA, Quesenberry CP, Sorel ME, Mendoza GR, Leong AB. Computer-based models to identify high-risk children with asthma. Am J Respir Care Med 1998; 157: 1173-80.

[5] Singh AK, Woodruff PG, Ritz RH, Mitchell D, Camargo CA. Inhaled corticosteroids for asthma: are ED visits a missed opportunity for prevention? Am J Emerg Med 1999; 17: 144-47.

[6] Stempel DA, Roberts CS, Stanford RH. Treatment patterns in the months prior to and after asthma-related emergency department visit. Chest 2004; 126: 75- 80 .

[7] Smith MJ, Rascati KL, McWilliams BC. Inhaled anti-inflammatory pharmacotherapy and subsequent emergency department visits among patients with asthma in the Texas Medicaid program. Ann Allergy Asthma Immunol 2004; 92: 40-6.

[8] Rodrigo GJ, Rodrigo C. Rapid-Onset asthma attack: A prospec-tive cohort study about characteristics and response to emergency department treatment. Chest 2000; 118: 1547-52.

[9] The National Health Interview Survey (NHIS) 2008 BRFSS Asthma Questions. Oct 27, 2009. Available from: http://www. cdc.gov/asthma/sur vey/brfss.html

[10] Camargo CA Jr, Clark S. Emergency department management of acute asthma between 1996-2001: potential impact of the 1997 NAEPP guidelines. J Allergy Clin Immunol 2002; 109(Suppl): S156.

[11] Cydulka R, Tamayo-Sarver J, Wolf C, Herrick E, Gress S. Inadequate follow-up controller medications in patients with asthma. Ann Emerg Med 2005; 24: 316-22.

(C) Lincourt et al.; Licensee Bentham Open.

This is an open access article licensed under the terms of the Creative Commons Attribution Non-Commercial License (http://creativecommons.org/licenses/ by-nc/3.0/) which permits unrestricted, non-commercial use, distribution and reproduction in any medium, provided the work is properly cited. 